\title{
Study of Molecular Interactions in Liquidmixture Using Ultrasonic Technique
}

\author{
Bhandakkar V. D. \\ Department of Electronics A.N. College, Warora - 442907
}

\begin{abstract}
The ultrasonic velocity, density and viscosity of the mixtures namely, methylmethacrylate with methanol, p-dioxane and cyclohexane at $303 \mathrm{~K}$ have been measured. From the measured data, some of the acoustical parameters such as adiabatic compressibility $\left(\beta_{a}\right)$, free length $\left(L_{f}\right)$, free volume $\left(V_{f}\right)$, internal pressure $\left(\pi_{i}\right)$, relaxation time $(\tau)$, acoustic impedance $(Z a)$ and Gibb's free energy $(\Delta G)$ values have been calculated for all the mixtures. The behavior of these parameters with composition of the mixture at the temperature $303 \mathrm{~K}$ has been discussed in terms of molecular interaction between the components of liquids.
\end{abstract}

Keywords: Adiabatic compressibility, free length, frees volume, internal pressure, molecular interactions and binary systems.

\section{Introduction:}

The study of inter molecular interactions plays an important role in the development of molecular sciences. In recent years ultrasonic technique has become powerful tool in providing information regarding the behavior of liquids and solids owing to its ability of characterizing physiochemical behavior of the medium. The study of miscibility and molecular interaction present in polymer like methylmethacrylate and solvent in a polymer solution is of great significance for engineering applications of polymers [1]. They also provide substantial information on the process involving polymer production and their uses [2, 3]. Further, polymer dissolution also plays a key role in many industrial applications in a variety of areas and an understanding of the dissolution process allows for the optimization of design and processing conditions as well as selection of suitable solvent $[4,5]$. Many researchers have undertaken these studies qualitatively through ultrasonic velocity, adiabatic compressibility and viscosity measurements [6-11]. However, there are very few studies on the quantitative study of the interactions in a polymer solution system $[\mathbf{1 2}, \mathbf{1 3}]$.

Methylmethacrylate (MM) is a versatile fluid and has been used on a wide range of fields and applications $[14, \mathbf{1 5}]$. In view of growing interest, in this paper, the results of an ultrasonic velocity, density and viscosity to study the related acoustical parameters, for the binary systems of methylmethacrylate + methanol, methylmethacrylate $+p$-dioxane and methylmethacrylate + cyclohexane at the temperature $303 \mathrm{~K}$ have been reported. The results are discussed in terms of molecular interactions.

2.1 Adiabatic compressibility ( $\square$ a :

\section{Theory:}

The adiabatic compressibility ( $\beta$ a) has been calculated from the ultrasonic velocity (U) and the density $(\rho)$ of the medium using the equation as:

$$
\beta \mathrm{a}=1 /\left(\mathrm{U}^{2} * \rho\right)
$$

2.2 Intermolecular free length $\left(L_{f}\right)$ :

Intermolecular free length has been determined as:

$$
L_{\mathrm{f}}=\mathrm{K}_{\mathrm{J}}\left(\beta_{\mathrm{a}}\right)^{1 / 2}
$$
liquid.

Where $K_{J}$ is the temperature dependent Jacobson's constant $[\mathbf{1 6 , 1 7 ]}$ but independent of the nature of

2.3 Free volume $\left(\mathrm{V}_{\mathrm{f}}\right)$ : liquid as:

The free volume $[15,17]$ has been calculated in terms of ultrasonic velocity $(U)$ and viscosity $(\eta)$ of the

$$
\mathrm{V}_{\mathrm{f}}=\left[\left(\mathrm{Me}_{\mathrm{ff}} \mathrm{U}\right) /(\mathrm{K} \eta)\right]^{3 / 2}
$$

Where, Meff (Effective mass) $=\Sigma \mathrm{mi} x i$, in which mi and xi are the molecular weight and the mole fraction of the individual constituents respectively. $\mathrm{K}$ is the temperature independent constant, which is equal to $4.28 * 10^{9}$ for all liquids and $\eta$ be the viscosity. 


\section{$2.4 \quad$ Internal pressure $(\square \mathrm{i})$ :}

On the basis of statistical thermodynamics, Suryanarayana [18], derived an expression for the determination of internal pressure $\left(\pi_{\mathrm{i}}\right)$ by the use of free volume concept as:

$$
\pi \mathrm{i}=(\mathrm{b} \mathrm{RT})[(\mathrm{K} * \eta) /(\mathrm{U})]^{1 / 2} *\left[\left(\rho^{2 / 3}\right) /\left(\mathrm{Me}_{\mathrm{ff}}{ }^{7 / 6}\right)\right] \quad-
$$

Where, $\mathrm{b}$ is the cubic packing which is assumed to be 2 for all liquids and solutions, $\mathrm{K}$ is the temperature independent constant, $\mathrm{T}$ is the absolute temperature, $\mathrm{R}$ is universal gas constant, $\eta$ be the viscosity and Meff the effective molecular weight .

\subsection{Relaxation time ( $\square$ ):}

Relaxation time ( $\square$ ) is the time taken for the excitation energy to appear as translational energy and it depends on temperature and on impurities. The dispersion of ultrasonic velocity in binary mixture reveals information about the characteristic time of the relaxation process that causes dispersion. The relaxation time ( $\square$ ) can be calculated from the relation as;
$\square=(4 / 3) \beta a * \eta$.
Where $\square \mathbf{a}$ and $\square$ are adiabatic compressibility and viscosity of the liquid and liquid mixtures.

\section{Acoustic impedance (Za):}

The specific acoustic impedance is given by

$\mathrm{Za}=\mathrm{U}_{*} \rho$.

Where $\mathbf{U}$ and $\square$ are the ultrasonic velocity and density of the liquid respectively.

\subsection{Gibb's free energy $(\square G)$ :}

The relaxation time for a given transition is related to the activation energy. The variation of relaxation time ( $\square$ ) with temperature (T) can be expressed in the form of Eyring self process theory.

$$
1 / \square=\left[\left(\mathrm{K}_{\mathrm{B}} \mathrm{T}\right) / \mathrm{h}\right] * \exp \left(-\Delta \mathrm{G} / \mathrm{K}_{\mathrm{B}} \mathrm{T}\right) \text {. }
$$

The above equation can be rearranged as,

$$
\Delta \mathrm{G}=\left(-\mathrm{K}_{\mathrm{B}} \mathrm{T}\right) \log \left[\mathrm{h} /\left(\mathrm{K}_{\mathrm{B}} \mathrm{T} \tau\right)\right] \text {. }
$$

$$
\text { or, } \quad \Delta \mathrm{G}=\left(\mathrm{K}_{\mathrm{B}} \mathrm{T}\right) \log \left[\left(\mathrm{K}_{\mathrm{B}} \mathrm{T} \tau\right) / \mathrm{h}\right] \text {. }
$$

Where $\mathbf{K}_{\mathbf{B}}$ is the Boltzmann's constant $\left(1.3806 \times 10 \square^{23} \mathrm{Jk}^{-1}\right)$, $\mathbf{h}$ is the plank's constant $\left(6.63 \times 10^{-34} \mathrm{JS}\right), \mathbf{T}$ be the absolute temperature and $\square$ be the relaxation time.

\section{Experimental Details:}

The component fluid namely methylmethacrylate (solute) and the methanol, p- dioxane and cyclohexane (solvents) were obtained of Anal R grade, again they were purified and redistilled in accordance with the usual procedure $[18,19]$ before use. The binary mixture of various mole fractions of two component in the systems, namely methylmethacrylate + methanol, methylmethacrylate + cyclohexane and methylmethacrylate $+p-$ dioxane (here after $\mathrm{p}$-dioxane referred as dioxane only), were prepared immediately before use.

The ultrasonic velocity $(\mathrm{U})$ of frequency $10 \mathrm{MHz}$ and density $(\rho)$ in these binary mixtures were measured by employing ultrasonic time inter- velometer(UTI -101, Innovative instrument, Hyderabad) and hydrostatic sinker method in the temperature range $10-40^{\circ} \mathrm{C}$, with an overall accuracy of $\pm 0.1 \mathrm{~m} / \mathrm{s}$, Ultra thermo state $\mathrm{U}-10$ maintained temperature of the sample constant to $\pm 0.1^{\circ} \mathrm{C}$. A specially designed and fabricated double walled metallic ultrasonic cell and glass cell along with 6 - digit monopan balance permitted to achieve accuracy of 1 in $10^{4} \mathrm{~m} / \mathrm{s}$ in velocity and 1 in $10^{4} \mathrm{gm}$ in density measurement. The viscosity $(\eta)$ was measured using Ostwald's viscometer with an accuracy of $\pm 0.0001 \mathrm{Nm}^{-2} \mathrm{~S}$. All the precautions were taken to minimize the possible experimental error. The setup is checked for standard liquids. The values obtained are compared with Literature [18-21] values and found that, they match very well with each other.

\section{TABLES}

Table - 4.1: Comparison of experimental values of density ( $\rho)$, viscosity $(\eta)$ and ultrasonic Velocity $(U)$ of pure liquids at $303 \mathrm{~K}$ with literature values:

\begin{tabular}{lcccccc}
\hline Pure liquids & \multicolumn{2}{c}{$\begin{array}{c}\text { Density }(\rho) \\
\left(\mathrm{Kg} \mathrm{m}^{-3}\right)\end{array}$} & \multicolumn{2}{c}{$\begin{array}{c}\text { Viscosity }(\eta) \\
\left(10^{-3} \mathrm{NSm}^{-2}\right)\end{array}$} & \multicolumn{2}{c}{$\begin{array}{c}\text { Velocity (U) } \\
(\mathrm{m} / \mathrm{s})\end{array}$} \\
& Expt. & Literature & Expt. & Literature & Expt & literature \\
\hline 1. Methylmethacrylate & 947.4 & --- & 0.4797 & --- & 1141.0 & ---- \\
2. Methanol & 776.1 & 777.7 & 0.5150 & 0.5030 & 1086.0 & 1087.6 \\
3. Dioxane & 1027.4 & 1028.1 & 0.8154 & 1.010 & 1324.0 & 1323.0 \\
4. Cyclohexane & 772.3 & 763.7 & 0.6887 & 0.68950 & 1240.0 & 1243.3
\end{tabular}


Study Of Molecular Interactions In Liquidmixture Using Ultrasonic Technique

Table - 4.2: The experimental values of density $(\rho)$, viscosity $(\eta)$ and Ultrasonic Velocity $(U)$ at $303 \mathrm{~K}$ for the systems - I, II \& III:

\begin{tabular}{|c|c|c|c|c|}
\hline \multicolumn{2}{|c|}{$\begin{array}{l}\text { Mole } \\
\text { Fraction }\end{array}$} & \multirow[t]{2}{*}{$\begin{array}{l}\operatorname{density}(\rho) \\
\quad\left(\mathrm{kg} \mathrm{m}^{-3}\right)\end{array}$} & \multirow[t]{2}{*}{$\begin{array}{l}\text { viscosity }(\eta) \\
\left(10^{-3} \mathrm{Nsm}^{-2}\right)\end{array}$} & \multirow[t]{2}{*}{$\begin{array}{l}\text { velocity } \\
\left(\mathrm{ms}^{-1}\right)\end{array}$} \\
\hline & $\mathrm{X} 2$ & & & \\
\hline \multicolumn{5}{|c|}{ System -I: methylmethacrylate + methanol. } \\
\hline 0.1 & 0.9 & 790.00 & 0.5380 & 1095.00 \\
\hline 0.2 & 0.8 & 807.20 & 0.5600 & 1101.00 \\
\hline 0.3 & 0.7 & 827.60 & 0.5830 & 1103.00 \\
\hline 0.4 & 0.6 & 835.10 & 0.6010 & 1112.00 \\
\hline 0.5 & 0.5 & 851.30 & 0.6240 & 1118.0 \\
\hline 0.6 & 0.4 & 867.30 & 0.6470 & 1123.00 \\
\hline 0.7 & 0.3 & 891.20 & 0.6700 & 1125.00 \\
\hline 0.8 & 0.2 & 899.30 & 0.6930 & 1133.00 \\
\hline 0.9 & 0.1 & 914.10 & 0.7160 & 1137.00 \\
\hline
\end{tabular}

System - II: methylmethacrylate + dioxane

$\begin{array}{rrrrr}0.1 & 0.9 & 1010.90 & 0.8088 & 1286.00 \\ 0.2 & 0.8 & 999.90 & 0.8022 & 1254.00 \\ 0.3 & 0.7 & 991.80 & 0.7957 & 1230.00 \\ 0.4 & 0.6 & 980.90 & 0.7890 & 1215.00 \\ 0.5 & 0.5 & 970.00 & 0.7824 & 1210.00 \\ 0.6 & 0.4 & 962.00 & 0.7757 & 1184.00 \\ 0.7 & 0.3 & 952.00 & 0.7691 & 1170.00 \\ 0.8 & 0.2 & 941.00 & 0.7623 & 1152.00 \\ 0.9 & 0.1 & 940.80 & 0.7588 & 1140.00\end{array}$

System - III: methylmethacrylate + cyclohexane

$\begin{array}{lllll}0.1 & 0.9 & 786.90 & 0.6947 & 1230.00 \\ 0.2 & 0.8 & 801.20 & 0.7007 & 1219.00 \\ 0.3 & 0.7 & 825.70 & 0.7068 & 1206.00 \\ 0.4 & 0.6 & 846.80 & 0.7130 & 1198.00 \\ 0.5 & 0.5 & 845.30 & 0.7193 & 1187.00 \\ 0.6 & 0.4 & 863.90 & 0.7253 & 1180.00 \\ 0.7 & 0.3 & 885.90 & 0.7314 & 1164.00 \\ 0.8 & 0.2 & 892.90 & 0.7376 & 1155.00 \\ 0.9 & 0.1 & 910.30 & 0.7437 & 1151.00\end{array}$

Table - 4.3: The values of adiabatic compressibility $(\beta \mathrm{a})$, free length $\left(L_{f}\right)$. Free Volume $\left(V_{f}\right)$, internal pressure $(\square i)$, Acoustic impedance $\left(Z_{a}\right)$, Relaxation time $(\tau)$ and Gibb's Free energy $(\Delta G)$ at $303 \mathrm{~K}$ for the systems: I, II \& III respectively.

\begin{tabular}{|c|c|c|c|c|c|c|c|c|}
\hline \multicolumn{2}{|c|}{\begin{tabular}{lc}
\multicolumn{2}{c}{ Mole Frac. } \\
$X_{1}$ & $X_{2}$
\end{tabular}} & $\begin{array}{c}\beta_{\mathrm{a}} \times 10^{-10} \\
\left(\mathrm{~m}^{2} \mathrm{~N}^{-1}\right)\end{array}$ & $\begin{array}{l}\mathrm{L}_{\mathrm{f}} \times 10^{-10} \\
(\mathrm{~m})\end{array}$ & $\begin{array}{c}\mathrm{V}_{\mathrm{f}} \times 10^{-7} \\
\left(\mathrm{~m}^{3} \cdot \mathrm{mole}^{-1}\right)\end{array}$ & $\begin{array}{l}\text { Лi x } 10^{6} \\
(\mathrm{~Pa} . \mathrm{S})\end{array}$ & $\begin{array}{l}\tau \times 10^{-12} \\
(\mathrm{~S})\end{array}$ & $\begin{array}{r}\mathrm{Zax} 10^{6} \\
\left(\mathrm{kgm}^{-2} \mathrm{~S}^{-2}\right)\end{array}$ & $\begin{array}{l}\Delta \mathrm{Gx} 10^{-20} \\
(\mathrm{KJ} m o l e\end{array}$ \\
\hline & & \multicolumn{7}{|c|}{ System- I: Methylmethacrylate + Methanol } \\
\hline 0.1 & 0.9 & 10.5371 & $0.6482^{\circ}$ & 0.7941 & 873.331 & 0.7573 & 0.8651 & 0.2841 \\
\hline 0.2 & 0.8 & 10.2192 & 0.6377 & 0.9606 & 846.642 & 0.7630 & 0.8887 & 0.2855 \\
\hline 0.3 & 0.7 & 09.9313 & 0.6287 & 1.1167 & 658.112 & 0.7719 & 0.9128 & 0.2876 \\
\hline 0.4 & 0.6 & 09.6794 & 0.6207 & 1.2970 & 980.623 & 0.7756 & 0.9286 & 0.2885 \\
\hline 0.5 & 0.5 & 09.2971 & 0.6083 & 1.4549 & 526.514 & 0.7734 & 0.9518 & 0.2880 \\
\hline 0.6 & 0.4 & 09.1422 & 0.6032 & 1.6071 & 482.992 & 0.7886 & 0.9740 & 0.2915 \\
\hline 0.7 & 0.3 & 08.8641 & 0.5940 & 1.7418 & 451.878 & 0.7918 & 1.0026 & 0.2922 \\
\hline 0.8 & 0.2 & 08.6623 & 0.5872 & 1.8993 & 417.522 & 0.8003 & 1.0189 & 0.2942 \\
\hline 0.9 & 0.1 & 08.4624 & 0.5803 & 2.2029 & 368.888 & 0.8078 & 1.0393 & 0.2959 \\
\hline
\end{tabular}


Study Of Molecular Interactions In Liquidmixture Using Ultrasonic Technique

\begin{tabular}{ccccccccc}
\hline \multicolumn{7}{c}{ System-II: Methylmethacrylate + dioxane } \\
0.1 & 0.9 & 05.9185 & 0.4854 & 1.9075 & 440.952 & 0.6382 & 1.3000 & 0.2531 \\
0.2 & 0.8 & 06.3591 & 0.5031 & 1.9006 & 434.666 & 0.6801 & 1.2539 & 0.2646 \\
0.3 & 0.7 & 06.6645 & 0.5150 & 1.9063 & 428.111 & 0.7070 & 1.2199 & 0.2717 \\
0.4 & 0.6 & 06.9059 & 0.5243 & 1.9328 & 419.371 & 0.7264 & 1.1918 & 0.2766 \\
0.5 & 0.5 & 07.0413 & 0.5294 & 1.9833 & 409.146 & 0.7345 & 1.1737 & 0.2786 \\
0.6 & 0.4 & 07.4105 & 0.5431 & 1.9820 & 403.730 & 0.7664 & 1.1397 & 0.2863 \\
0.7 & 0.3 & 07.6735 & 0.5527 & 2.0094 & 395.616 & 0.7868 & 1.1138 & 0.2911 \\
0.8 & 0.2 & 08.0051 & 0.5645 & 2.0267 & 388.311 & 0.8136 & 1.0844 & 0.2972 \\
0.9 & 0.1 & 08.1782 & 0.5708 & 2.0461 & 383.093 & 0.8273 & 1.0725 & 0.3002 \\
& & & & & & & & \\
& & & System III: methylmethacrylate + Cyclohexane & & 0.9679 & 0.2890 \\
0.1 & 0.9 & 8.3998 & 0.5782 & 2.1131 & 370.744 & 0.7780 & 0.9767 & 0.2906 \\
0.2 & 0.8 & 8.3995 & 0.5582 & 2.1156 & 370.502 & 0.7847 & 0.9767 \\
0.3 & 0.7 & 83269 & 0.5757 & 2.1157 & 371.0 & 0.7847 & 0.9958 & 0.2903 \\
0.4 & 0.6 & 8.2282 & 0.5723 & 2.1191 & 375.141 & 0.7822 & 1.0145 & 0.2900 \\
0.5 & 0.5 & 83963 & 0.5781 & 2.1175 & 370.441 & 0.8052 & 1.0034 & 0.2953 \\
0.6 & 0.4 & 8.3133 & 0.5752 & 2.1270 & 371.009 & 0.8039 & 1.0194 & 0.2950 \\
0.7 & 0.3 & 8.3312 & 0.5758 & 2.1119 & 394.186 & 0.8124 & 1.0312 & 0.2969 \\
0.8 & 0.2 & 8.3952 & 0.5780 & 2.1264 & 371.787 & 0.8256 & 1.0313 & 0.2998 \\
0.9 & 0.1 & 8.2588 & 0.5733 & 2.1243 & 371.687 & 0.8189 & 1.0478 & 0.2984 \\
& & & & & & & & \\
\hline
\end{tabular}

\section{Result discussion:}

The experimentally measured and literature values [18-20] of density, velocity and viscosity for pure liquids at $303 \mathrm{~K}$ are presented in table -4.1. Experimental density, viscosity and ultrasonic velocity values for the three binary systems namely, system-I: methylmethacrylate + methanol, system-II: methylmethacrylate +dioxane and system-III : methylmethacrylate+ cyclohexane respectively at $303 \mathrm{~K}$ are given in the table 4.2. The parameters, adiabatic compressibility $\left(\beta_{\mathrm{a}}\right)$, free length $\left(\mathrm{L}_{\mathrm{f}}\right)$, free volume $\left(\mathrm{V}_{\mathrm{f}}\right)$, acoustic impedance $(\mathrm{Za})$, internal pressure $(\pi \mathrm{i})$, relaxation time $(\tau)$ and Gibb's free energy $(\Delta \mathrm{G})$ at temperature $303 \mathrm{~K}$ are listed in table 4.3. The variation of $\beta \mathrm{a}, \mathrm{L}_{\mathrm{f}}$ and $\mathrm{Vf}, \mathrm{Vs}$ mole fraction $(\mathrm{x})$ at $303 \mathrm{~K}$ for the system -I : methylmethacrylate+methanol, system- II : methylmethacrylate+ dioxane \& system III - methylmethacrylate+ cyclohexane are shown in the fig. $\mathbf{1 , 2}$ and 3 respectively. The variation of $\pi \mathrm{i}, \mathrm{Za}, \tau$ \& $\Delta \mathrm{G}$ Vs mole fraction(x) at $303 \mathrm{~K}$ for the system - I: methylmethacrylate + methanol, system-II: methylmethacrylate+ dioxane and system -III methylmethacrylate + cyclohexane are shown in the fig. $4, \mathbf{5 , 6} \& \mathbf{6}$ respectively.

From the table - 4.2, it is noted that, the density $(\rho)$ increases with increase in mole fraction for the systems-I \& III i.e. for methylmethacrylate + methanol and methylmethacrylate + cyclohexane and the density decreases for the system -II i.e. for the system methylmethacrylate+ dioxane with increase in mole fraction. Ultrasonic velocity and viscosity increases with increase in mole fraction of the solute in case of the system - I (methylmethacrylate + methanol). In case of the system -II (methylmethacrylate +dioxane) ultrasonic velocity

and viscosity decreases with increase in mole fraction of the solute. While in case of the system- III (methylmethacrylate + cyclohexane), ultrasonic velocity decreases, and viscosity increase with increase in mole fraction of the solute. The increase in the velocity, in the system- I (methylmethacrylate +methanol) is due to decrease in adiabatic compressibility and free length (fig-1 \& fig-2). In the system -II (methylmethacrylate+dioxane), velocity decreases, due the increase in adiabatic compressibility and the free length (fig- $1 \&$ fig- 2).

It has been observed that for a given concentration, as the number of $\mathrm{CH}$-group or Chain length changes, the sound velocity changes. This may lead to the presence of specific molecular interaction between the molecules of the liquid mixture. The adiabatic compressibility and free length are the deciding factors of the ultrasonic velocity in the liquid systems. The internal pressure decrease (fig-4) and free volume increase (fig-3) with increasing mole fraction of the salute in all the three systems. The internal pressure may gives information regarding the nature and strength of forces existing between the molecules. It represents the presence of weak interaction between the solute and solvent molecules.

Acoustic impedance (Za) increases with increase in the mole fraction of the solute in the systems- I \& III and decrease in the system- II (fig-6). The relaxation time $(\tau)$ also increases with increasing the mole concentration of the solute in all the systems (fig-5). The dispersion of the ultrasonic velocity in the system may contain information about the characteristic time $(\tau)$ of the relaxation process that causes dispersion. The relaxation time which is in the order of $10^{-12}$ sec., is due to structural relaxation process [22] and in such a situation, it is suggested that, the molecules get rearranged due to co-operative process [23]. 
The Gibb's free energy $(\Delta G)$ increases with increasing mole fraction the solute of all the systems (fig-7). This may be due to the intermediate compound formation between the binary liquids. It is observed that, generally, the increase in Gibb's free energy favors the formation of product from reaction. This observation confirms the formation of hydrogen bonding in the binary mixture.
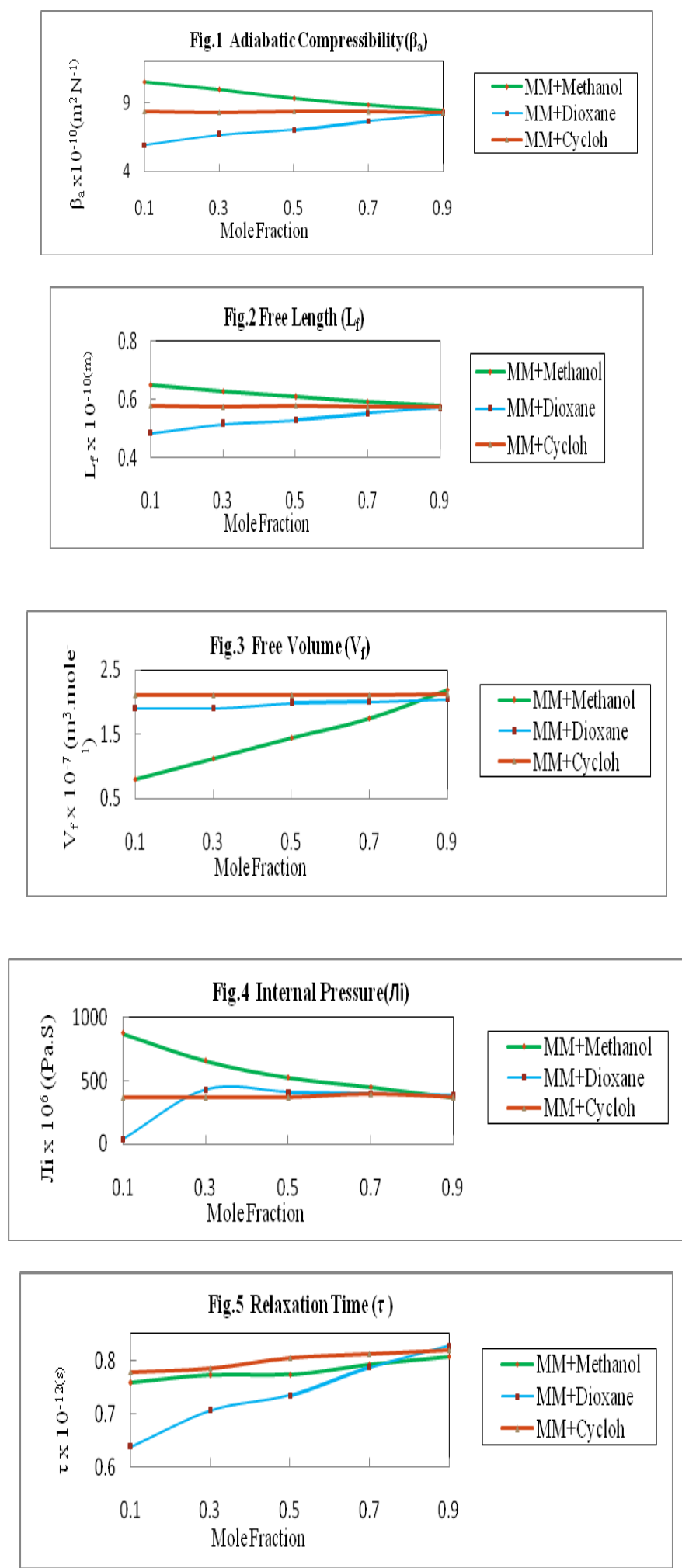

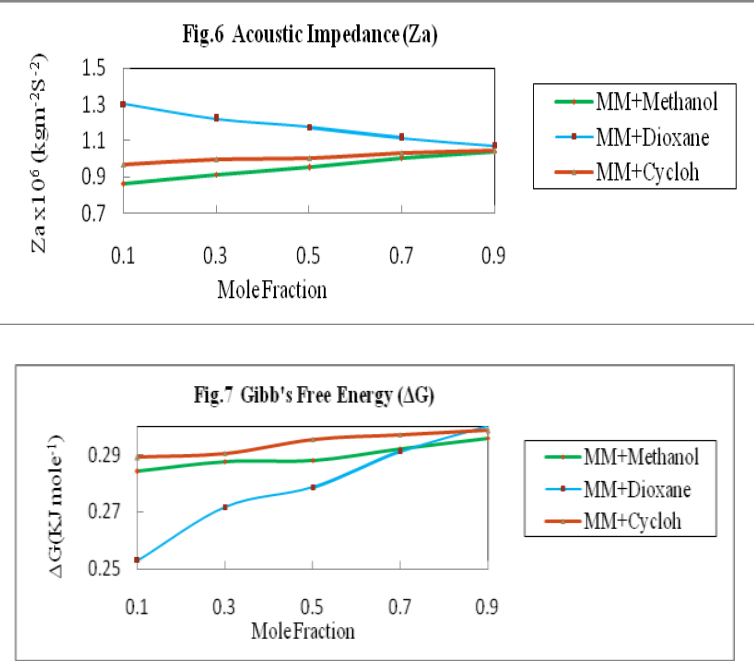

\section{Conclusion:}

The molecular interactions present in methylmethacrylate with methanol, dioxane \& cyclohexane as a solvent have been studied by viscosity, density and ultrasonic velocity study. Polymer solvent interactions parameters for the systems have been estimated based on Gibb's free energy calculations using data on viscosity and ultrasonic velocity of the solutions, at $303 \mathrm{~K}$ temperature. The result indicates the existence of molecular interaction between the polymer and the solvent in their solutions. The result is also shows the presence of higher degree of molecular interaction between methylmethacrylate and methanol in solution compared to methylmethacrylate with dioxane and cyclohexane in solutions.

The computed acoustical parameters and their values show the presence of specific molecular interaction in the mixtures. Hence it is concluded that, the association in this mixture is the result of Hydrogen bonding in the binary liquid mixtures. The dependence of ultrasonic velocity and other derived parameters on composition of the mixtures is indication of the presence of molecular interactions. The interaction primarily of dipoledipole and dipole -induce dipole type and becomes stronger with mole-fraction in methylmethacrylate + methanol system.

\section{Acknowledgement:}

The author, VDB ([File No.: F-47-919/09(WRO)]) is very much thankful to the university Grant commission for providing financial assistance in the form of minor research project (MRP).

\section{References:}

[1] Paul D R \&Newman's, Polymer Blends, Acad.Press, New, New York, vol.1\&2,(1978), 175

[2] Tompa H., Polymer solutions, Butterworth scientific, London, (1956), 215.

[3] Beth A M C and Jack L.K., Prog. Polym. Sci, 28,(2003), 1223.

[4] M Selvakumar \& Krishna Bhat, Ind.Jour.of Pure and appl. Phy, 46, (2008), 12-718.

[5] C Shanmuga Priya, et.al.International Jour. of adv. Sci. and Tech., (2010), 56-73.

[6] T Sumathi et al, Indian Journal of Pure and applied Physics, 49 (2011) 328-334.

[7] Bhandakkar V D, Tabhane V A \& Sharda Ghose, Indian Journal Pure and applied Physics, 41, (2003), 849 -854.

[8] Bhandakkar V D, Advances in Applied Science and Research Journal, Pelagia Research Library, 2(3), (2011), $198-207$.

[9] Bhandakkar V D et al, Journal of Chemical and Pharmaceutical Research, 2(4), (2010), 873-877.

[10] Bhandakkar V D et al, Indian Journal Pure Applied Physics, 49, (2011), 550-5523.

[11] Bhandakkar V D, Bedre G R, Suryavanshi , Advances in applied Science ResearchJournal, Pelagia research Library, 2 (4), (2011), 380-386.

[12] Kalyanasundaram S, et al, J Polym, Mater, 14, (1997), 285.

[13] Seetharaman V et at, J Mol Liq, 121, (2005), 156.

[14] Yang H, et al, J Mater Chem. Phy, 110, (2008), 38.

[15] Dai H et al, Electrochemical Acta, 53, (2008), 5113.

[16] Jacobson B, J Chem. Phys, 20, (1952), 927.

[17] Jacobson B, Acta Chem, Scand, 5, (1957), 1214, 6, (1952).

[18] Suryanarayana C V, J. Acoustic soc. India, 7, (1979), 131.

[19] Vogel A I, A Text book of Practical Organic chemistry, Longman group Limited, London, (1956)

[20] J. Timmerman, Physico- Chemical constants of Pure Organic Compounds, Elsevier, New York (1950).

[21] Thiyagarajan R \& Palaniappan L, Indian Journal of Pure \& applied Physics, 46, (2008), 852- 856.

[22] Kinsler L E \& Rray A R, Fundamentals of Acoustics (Wiley eastern), New Delhi (1989).

[23] Ali A Hyder S and Nair A K, Indian J. Pure \& Appl. Phy., 7413, (2000), 63. 